\title{
Exploiting Spectrum Spatial Reusability for Routing in Multi-hop Wireless Networks ${ }^{\star}$
}

\author{
Tong Meng and Fan $\mathrm{Wu}^{\star \star}$ \\ Shanghai Key Laboratory of Scalable Computing and Systems, \\ Department of Computer Science and Engineering, \\ Shanghai Jiao Tong University, China \\ \{mengtong, fan-wu\}@sjtu.edu.com
}

\begin{abstract}
Routing metrics are essential to achieve a good end-to-end throughput on a min-cost path in multi-hop wireless networks. Originally, most routing algorithms were based on min-hop count metric. Then link-quality aware metrics, ETX and ETT, are proposed. After that, the emergence of opportunistic and any-path routing, leads to metrics such as EOTX and EAX. But almost all the previous routing metrics take the sum of all the link weights when choosing a min-cost path. They ignore that a pair of links on a path might be able to transmit at the same time without interference and hence it is inappropriate to include both of their weights in the whole path. In this work, we suggest exploiting such spatial reusability when designing routing metric to improve end-to-end throughput. We propose Spatial Reusability Aware Routing Metric (SAR) with the corresponding Spatial Reusability Aware Routing Algorithm. Our simulation results have shown good improvement in throughput.
\end{abstract}

Keywords: Spatial Reusability, Routing, Multi-hop, Wireless Network.

\section{Introduction}

In wireless network, it is essential to find the best path between data source and destination to achieve high end-to-end throughput, which is the task of routing algorithms. Originally, most routing algorithms were based on min-hop count metric, which is a metric that assumes perfect wireless links and tends to minimize the number of hops on the path. However, in the face of lossy links in wireless environment, protocols using min-hop metric does not perform well because they may include some poor links with high loss ratios. Then taking link quality into consideration, a lot of link-quality aware metrics have been designed. De Couto et al. [1] proposed the Expected Transmission Count (ETX)

\footnotetext{
^ This work was supported in part by China NSF grant 61170236 and 61133006 . The opinions, findings, conclusions, and recommendations expressed in this paper are those of the authors and do not necessarily reflect the views of the funding agencies or the government.

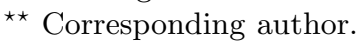

X. Wang et al. (Eds.): WASA 2012, LNCS 7405, pp. 670-676, 2012.

(C) Springer-Verlag Berlin Heidelberg 2012 
that weights the links by the expected number of retransmissions depending on their loss ratios. With link quality measured more precisely, ETX-based routing algorithms improves the end-to-end throughput significantly. Inspired from ETX and by adding the factor of link bandwidth, Draves et al. 22 developed the Expected Transmission Time (ETT) and got even better performance. After that, the emergence of new ideas of routing, namely opportunistic and any-path routing, leads to metrics such as EOTX [3] and EAX [4].

We notice that almost all the previous routing metrics, ETX as an example, simply take the sum of all the link weights when choosing a min-cost path. This method seems reasonable when all links on a path are in each other's interference range. Yet for a long path, a pair of links on it might be able to transmit at the same time without interference and hence it is inappropriate to include both of their weights in the whole path. We regard this phenomenon as a form of spatial reuse.

In this work, we suggest exploiting spatial reusability of wireless communication media in multi-hop wireless networks to improve end-to-end throughput. We also propose a simple routing metric called Spatial Reusability Aware Routing Metric (SAR) taking spectrum spatial reusability into account and develop corresponding Spatial Reusability Aware Routing Algorithm to implement SARmetric. Our main contributions are that we introduce a new idea in designing routing metric which is exploiting spectrum spatial reusability, and bring chance to further improve end-to-end throughput of multi-hop wireless networks.

Our simulations on ns-2 have shown strong evidence of the existence and effect of "spatial reuse" on a path. We test our algorithm in a 80-node network with a modified AODV protocol and compare the throughputs achieved with our metric on different paths with that with the original ETX metric. The results show that about $10 \%$ of all the source-destination pairs achieve apparent improvement with an average of nearly $400(\mathrm{kbps})$.

\section{System Model and Motivating Example}

In this section, we illustrate the network model we employ and provide motivating example to demonstrate the necessity of considering "spatial reusability" in routing metric.

\subsection{System Model}

As most of the other works in routing metrics, we consider a static multi-hop wireless network, in which each of the nodes has a single radio interface and works on the same channel. In this preliminary work, we focus on deterministic singlepath routing. In our following works, we will consider the case of opportunistic any-path routing.

We assume the loss ratio of the link is determined by SINR/BER model. As in (1), the SINR value is the received signal strength ratio between the packet that's being accessed $P$, and the addition of noise $N$ and interference packets $I$. 


$$
S I N R=\frac{P}{N+I} .
$$

According to the SINR/BER table, we can get the bit error rate of the packet from its SINR value. The loss ratio $r$ is calculated by (2) using the packet size $l$. In that way, whether a packet can be successfully received by a node is determined by the packet's SINR.

$$
r=1-(1-B E R)^{l} .
$$

We also assume every transmission is independent with identical distribution of loss ratio. To exploit the spatial reusability, two hops in a network can transmit free of interference if their source and destination nodes are at least out of each other's interference range (IR). With the network model we explain in the above, we show an inspiring example that demonstrates the power of spatial reusability as follows.

Because we only consider single-rate, single-radio case currently, ETX is applied to evaluate the weight of each wireless link. The ETX of a link is calculated from the loss ratios in the forward and reverse directions. Denote by $r_{f}$ and $r_{r}$, the forward and reverse delivery ratios of a link is the probabilities that a data packet and an ACK packet can be successfully received, respectively. The expected number of transmissions is [1]:

$$
\operatorname{ETX}=\frac{1}{\left(1-r_{f}\right) \times\left(1-r_{r}\right)} .
$$

The equation (3) is based on the assumption that the transmission of a packet can be regarded as a Bernoulli trial.

\section{$2.2 \quad$ Motivating Example}

To show the effect of spatial reusability in wireless routing, let's consider two paths as shown in Fig. 1. In Fig. 1, there're two routes between source node 1 and destination node 6 . They are route I with 5 hops and route II with 4 hops. The ETX value of each hop is indicated beside the links in the figure. Note that the first and the last hop on route I can work at the same time without interfering each other, while there're no such link pair on route II. For convenience, we define such kind of link pair to be a reuse pair.

By the ETX metric, route II is preferred because its ETX sum, which is 10.1, is smaller than the value 11.1 of route I. However, considering the fact that link (1-2) and (5-6) can work in the meanwhile, they together do not need to consume as much bandwidth as two consecutive hops. Thus if we subtract the weight of link (5-6) from route I's ETX, it will get smaller ETX than route II instead; and by estimation, route I can achieve a $10 \%$ higher throughput than route II because the first four hops on route I have better quality compared with the hops on route II. 


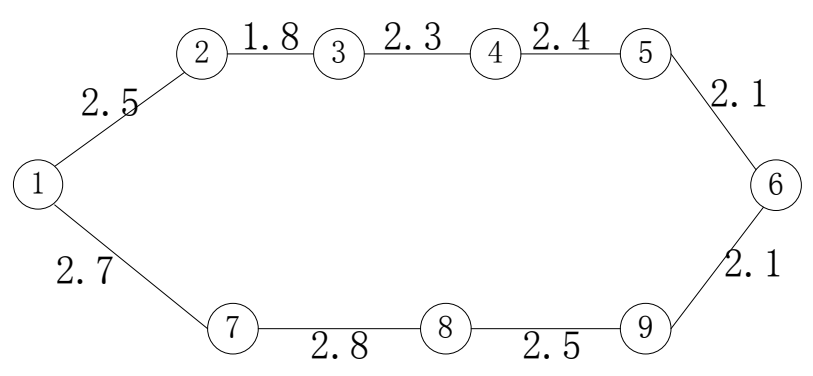

Fig. 1. A motivating example showing the effect of spatial reuse

Fig. 1 demonstrates the raw ETX metric's drawback of ignoring the possibility of "spatial reusability" between links. Taking such reusability into consideration, we give our simple metric in the next section.

\section{SAR Algorithm}

In this section, we explain our SAR Metric and present the SAR Algorithm based on the metric. Different from ETX which simply sums up the ETX values of all the links on a path, in SAR metric we consider the combination of independent units (IUs), which is a group of links where every two of them can make up a reuse pair. An IU can also contain only one single link which does not belong to any reuse pairs. We use $W_{i}$ to denote the weight of the $i$-th IU whose value is equal to the largest weight among all the links of an IU. In our Metric, the weight of the path, represented by $W_{\text {path }}$, is calculated by equation (4).

$$
\mathrm{W}_{\text {path }}=\sum_{i}\left(\mathrm{~W}_{i}\right)
$$

We only count the weight of IUs in our metric. So those hops from the same IU will be counted only once. In that way, spatial reusability between links in wireless networks can be synthesized in the design of routing metric. Now the main task of the metric will be deciding the combination method of independent units on a path. Because find an optimal combination of IUs, which is similar to the problem of determining the chromatic number of a graph in graph-coloring field, is known to be NP-complete, we suggest finding sub-optimal routes using one of the many existing polynomial time graph-coloring algorithms. We apply a simple first-fit algorithm to decide IU combination and calculate the weight of a path shown as below.

The SAR Algorithm goes through all the links from source to destination, and judges if a link is in the same independent unit with some link before it on the path. If so, add this link to that unit and update the IU's weight to be the biggest weight among all its links. Otherwise, create a new IU consisting of this single link. Hence the algorithm is in $O\left(n^{2}\right)$ and ends in polynomial time. 


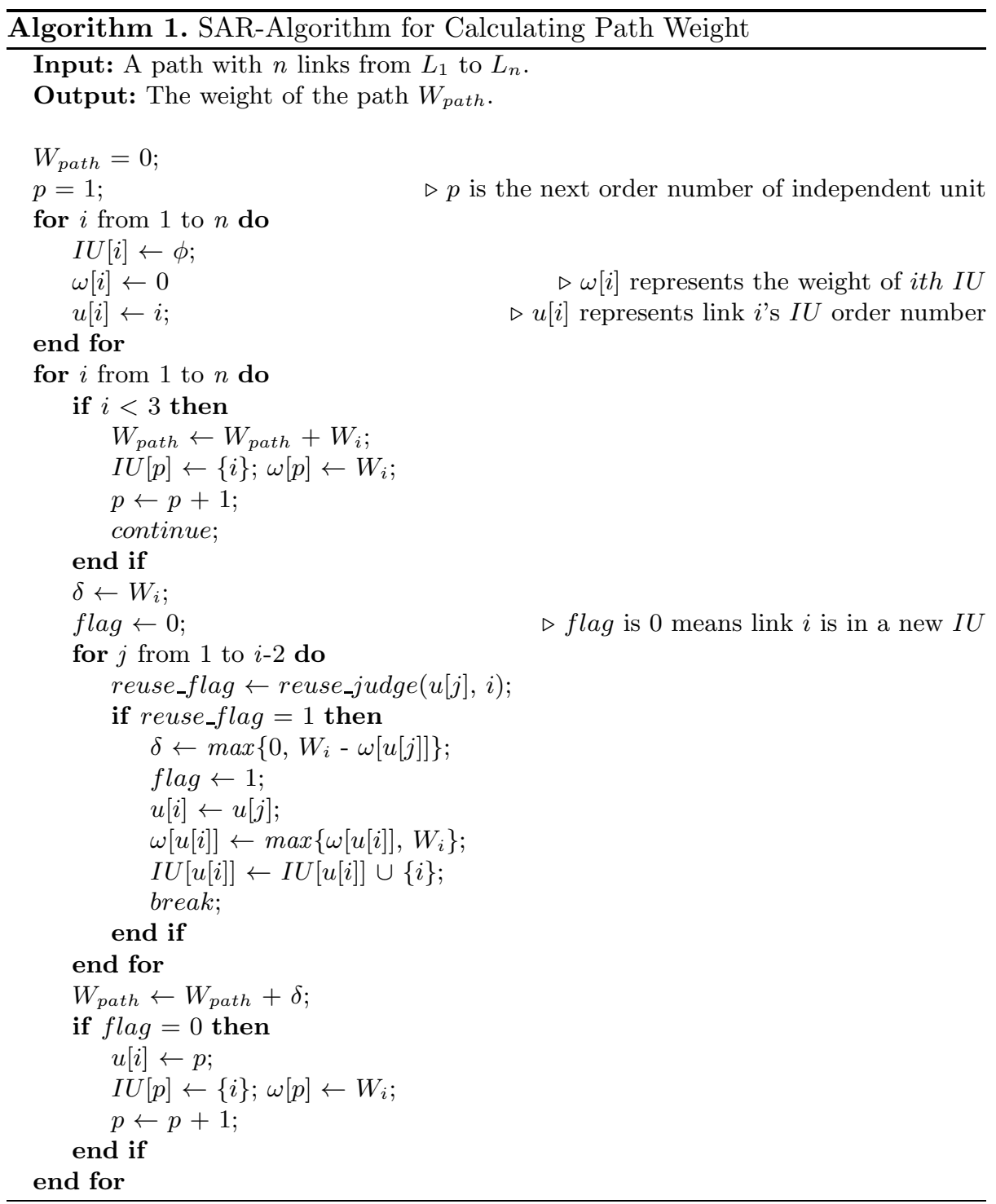

Plus the number of links on a path won't be quite huge, our SAR algorithm won't bring too much overhead to the routing process. Note that the function reuse_judge $(x, y)$ on the above is to judge whether link $y$ belongs to independent unit $x$. If the link can reuse with all the links from the unit, the function returns 1 ; or it returns 0 . 


\section{Implementation}

In this preliminary work, a modified AODV protocol with additional reuse information header is implemented to integrate SAR Algorithm. What's more, we take the real scene of transmitting in wireless environment into consideration and add an interference factor to SAR Metric. This is because in the original design of our metric, the weight of a IU is the largest weight among all links in that unit and the other links' weights are then omitted, whereas that's in the case where the links of the same IU can actually work at the same time. Without link-level synchronization, two links from the same unit may not transmit simultaneously sometimes. That results in interference between IUs and may lead to more transmissions before a packet is successfully received by the destination. So we add the interference factor to counteract the phenomenon whose value is $10 \%$ of the unit's weight. Every time a new hop is grouped into an independent unit, we add such a factor to the weight of the whole path. We calculate the factor that way to reflect the fact that the kind of interference is related to the weight of the IU to which the new hop belongs. What's more, because we only consider single rate case currently, ETX is stilled applied to evaluate the weight of each wireless link.

\section{Simulation}

In this section, we compare SAR implemented as in the above section with the original ETX-based AODV using simulations on ns-2. We build a network with 80 nodes within an $2000 \mathrm{~m} \times 2000 \mathrm{~m}$ area. RTS/CTS is turned off and CBR is used to generate 1500-byte packets under a $11 \mathrm{M}$ bandwidth. For simulations, 150 source and destination pairs are chosen, all of which are at least $900 \mathrm{~m}$ and at most $1200 \mathrm{~m}$ away. Furthermore, for every single source destination pair, we choose to use the original path found by ETX if weight of the best path under SAR is smaller than that of the original one by less than $10 \%$.

Fig. 2 plots all these paths' throughputs. The $x$ value of all the points is the throughput from ETX-based AODV. The $y$ value is the result of the same pair of source and destination from SAR. Those nodes above link $\mathrm{y}=\mathrm{x}$ correspond to those paths where SAR outperforms original ETX. From the figure, we can see that about $10 \%$ of all the simulated pairs achieve apparent throughput improvement compared with original ETX. The average improvement of them is calculated to be 375.98 (kbps), and we notice that there're several nodes that get almost $100 \%$ higher throughput among them and one data point shows even $200 \%$ improvement. There're still a larger part of all the source-destination pairs that performs equally under the two metrics. Some of those pairs use the same path because of our "at least $10 \%$ better" restriction. Still there are groups that choose different paths from the two metrics. That can also prove that integrating spatial reusability in the design of routing metric does not impair the network's performance. 


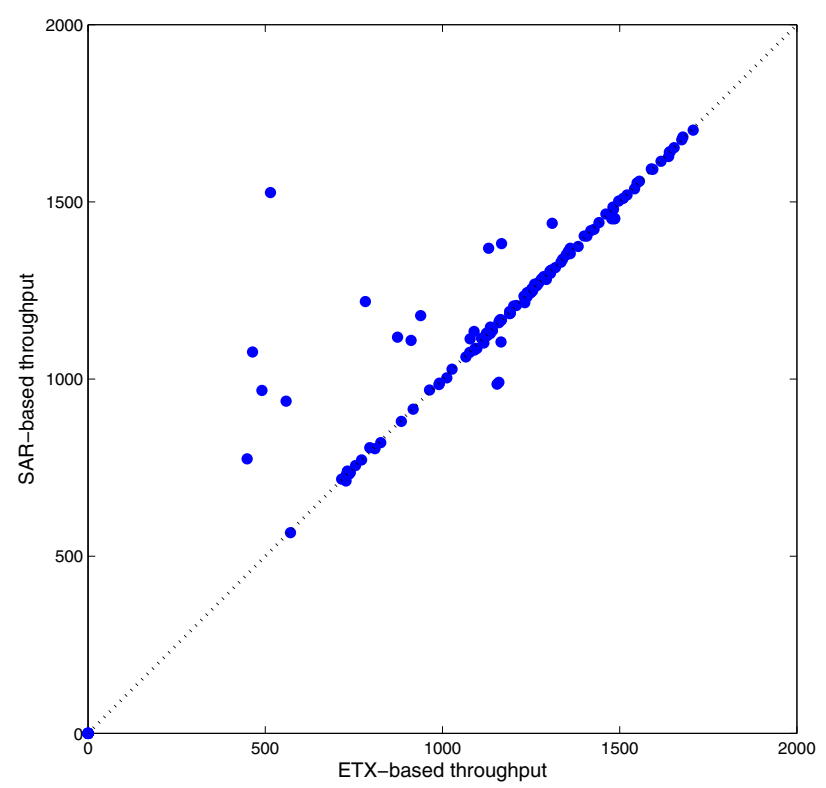

Fig. 2. Throughput comparison of two metrics

\section{Conclusion}

In this work, we have proposed to exploit the spatial reusability in wireless multihop routing and design a simple routing metric called SAR and an algorithm as well. Our simulation results demonstrate the possibility of improving the throughput by exploiting spectrum spatial reusability in wireless networks. In the future, we intend to investigate effective algorithms for counting reuse pairs. Opportunistic any-path routing is to be extended, too.

\section{References}

1. De Couto, D., Aguayo, D., Bicket, J., Morris, R.: A High-throughput Path Metric for Multi-Hop Wireless Routing. In: ACM MOBICOM (2003)

2. Draves, R., Padhye, J., Zill, B.: Routing in Multi-radio, Multi-hop Wireless Mesh Networks. In: ACM MOBICOM (2004)

3. Chachulski, S.: Trading Structure for Randomness in Wireless Opportunistic Routing. M.S. Thesis (2007)

4. Zhong, Z., Wang, J., Nelakuditi, S., Lu, G.-H.: On Selection of Candidates for Opportunistic Opportunistic Anypath Forwarding. In: ACM SIGMOBILE (2006) 